$$
\text { "hannula" — 2007/2/15 — 13:51 — page } 317 \text { — \#1 }
$$

\title{
Levels of students' understanding on infinity
}

\author{
Markku S. Hannula, Erkki Pehkonen, \\ Hanna Maijala and Rittta Soro
}

\begin{abstract}
Here we report some results of a two-year study for grades 5-6 and 7-8 (during the academic years 2001-03). The study included a quantitative survey for approximately 150 Finnish mathematics classes out of which 10 classes were selected to a longitudinal part of the study. Additionally, 40 students from these classes participated also a qualitative study. This paper will focus on students' understanding of infinity and the development of that understanding. The results show that most of the students did not have a proper view of infinity but that the share of able students grew, as the students got older.
\end{abstract}

Key words and phrases: infinity, metaphors, conceptual change, understanding.

ZDM Subject Classification: C33.

Most primary children are very interested in infinity, and they enjoy discussing the concept, if the teacher is only ready for it. On one hand, they have a concrete view on the world around and mathematics and, on the other hand, they are ready to play with numbers. Thus, questions on infinity may also come into light. In the primary curriculum, the concept 'infinity' is implicitly present in many of the topics, e.g. in arithmetic, when dealing with fractions, or in geometry, e.g. when introducing the concept 'straight line'. Infinity awakes curiosity in children already before they enter school: "preschool and young elementary school children show intuitions of infinity" [24]. However, this early interest is not often met by school mathematics curriculum, and infinity remains mysterious for most students throughout school years.

Copyright (C) 2006 by University of Debrecen 


$$
\text { "hannula" — 2007/2/15 — 13:51 — page } 318 \text { — \#2 }
$$

\section{Infinity in mathematics}

Actual and potential infinity

Infinity is an important concept in mathematics, especially in analysis, and we encounter infinity already in, perhaps, the most basic of mathematical concepts, namely in natural numbers. Consider the sequence of natural numbers $1,2,3, \ldots$ and think of continuing it on and on. There is no limit to the process of counting; it has no endpoint. Such ongoing processes without an end are usually the first examples of infinity for children; such processes are called potentially infinite.

In mathematics, such unlimited processes are quite common. Consider, for example, drawing regular polygons with more and more sides inside a circle, or counting more and more decimals of $\pi$. However, the interesting cases in mathematics are, when infinity is conceptualised as a realised "thing" - the socalled actual infinity. The set of all natural numbers is an example of actual infinity, because it requires us to conceptualise the potentially infinite process of counting more and more numbers as if it was somehow finished [11].

The question of infinity has its roots already in the mathematics of ancient Greece, cf. for example, the famous paradox of Zenon [2]. However, the transition from potential to actual infinity includes a transition from (an irreversible) process to a mathematical object. This step Greek mathematicians were unable to accomplish [16]. In the history of mathematics, the exact definition of and dealing with infinity is about one hundred years old. The foundation of infinity as modern mathematics sees it was laid when Dedekind and Cantor solved the problem of potential infinity at the end of the 19. century, and Cantor developed his theory of cardinal numbers (e.g. [2], [16]).

We may distinguish different kinds of infinities in mathematical objects. For example, the set of natural numbers has infinitely many elements, and it has no upper bounds. Therefore, the numbers may become bigger and bigger. Whereas, some sub-sets of rational numbers are different. For example, the set of rational numbers between zero and one also has infinitely many elements, but it is bounded. Furthermore, between any two rational numbers (however close they are to each other) there are infinitely many rational numbers. This property of rational numbers is called density.

When mathematicians write about counterintuitive nature of infinity, they typically refer to actual infinity. For example, take the (actually infinite) set of natural numbers $\{1,2,3, \ldots\}$ and compare it with its subset which includes only all even numbers $\{2,4,8, \ldots\}$. Intuitively, one would assume that the "number 


$$
\text { "hannula" — 2007/2/15 — 13:51 — page } 319 \text { — \#3 }
$$

of elements' in the latter would be smaller by half. However, the concept "equal number of elements" in a set is generalized to infinite sets by saying that the two sets have "equal number" of elements, if their elements can be put into one-to-one correspondence with each other; thus we say that their cardinality is the same (e.g. [2]).

Tsamir and Dreyfus [22] summarise the problems mathematicians have had with actual infinity, as follows:

Actual infinity, a central concept in philosophy and mathematics, has profoundly contributed to the foundation of mathematics and to the theoretical basis of various mathematical systems. It has long and persistently been rejected by mathematicians and philosophers alike, and was highly controversial even in the last century in spite of the comprehensive framework provided for it by Cantorian set theory.

They also cite Davis \& Hersh who warned that "the infinite has turned out to be the hiding place of much that is strange and paradoxical" ([4, p. 155]), and Fishbein [7] who explained that

when dealing with actual infinity - namely with infinite sets - we are facing situations which may appear intuitively unacceptable ... we are intuitively not equipped to deal with actually given infinite sets. Their logic is not our logic, which is rooted in our practical experience ([7, p. $92])$.

Hence, although the concept of infinity as a potentiality is relatively easy for mathematicians, the concept of actual infinity is counterintuitive and difficult.

\section{Students' conceptions of infinity}

Infinity has been an inspiring, but difficult concept for mathematicians. It is no wonder, that also students have had difficulties with it, although they might be fascinated about it. Previous research has identified typical problems and constructive teaching approaches to cardinality of infinite sets. Students use intuitively the same methods for the comparison of infinite sets as they use for the comparison of finite sets. Although students have no special tendency to use the Cantorian method of "one-to-one correspondence", they are prone to visual cues that highlight the correspondence. For example, students tend to match the set $\{1,2,3, \ldots\}$ more easily with the set $\left\{1^{2}, 2^{2}, 3^{2}, \ldots\right\}$ than with the set $\{1,4,9, \ldots\}$ $[22]$. 


$$
\text { "hannula" — 2007/2/15 - 13:51 — page 320 — \#4 }
$$

One issue that was raised already by Piaget [19], is the cognitive development of grasping infinitely large and infinitely dense. Wheeler [24] considered these questions, as follows:

Our decimal numeration system provides two perspectives of infinity: counting leads to a consideration of numbers that continue to get bigger without bounds, whereas successive partitionings of an interval lead to a consideration of numbers that continue to get smaller and are bounded.

Does a hierarchical relationship exist between understanding the decimal system and understanding of the other? [24]

Wistedt and Martinsson [25] have challenged Swedish $5^{\text {th }}$ graders (11 years old) with a realistic problem of dividing a one meter long piece of wood into three equal parts. Mathematically, the task can be reduced to dividing 100 with 3 . Since the students could only think of finite or potentially infinite decimals, they obtained either numbers that are not exactly equal or their sum is not exactly 100.

Fishbein, Tirosh and Hess inquired students' view of infinite partitioning through using successive halving of a number segment [8]. They concluded that students on grades 5-9 seem to have a finitist rather than a nonfinitist or an infinitist point of view in questions of infinity.

Even at the university level, the concept of infinity of real numbers is not clear for all students [15]. For example, Wheeler [24] points out that university students distinguished between $0.999 \ldots$ and 1 , because "the three dots tell you the first number is an infinite decimal".

\section{Metaphorical thinking}

\section{Metaphorical thought}

The view exposed here is based on an understanding of mathematical thinking as embodied [11]. Embodiment of mathematical thinking means an acceptance of biological grounding of thinking in human brain, an effort to accept and adopt results from neurosciences to human thinking. One important cognitive process that is well grounded in modern cognitive science and that can explain a lot of mathematical thinking is metaphorical thought.

"For the most part, human beings conceptualize abstract concepts in concrete terms, using ideas and modes of reasoning grounded in the sensorymotor system." ([11, p. 5]) 


$$
\text { "hannula" — 2007/2/15 - 13:51 — page 321 — \#5 }
$$

This process is called conceptual metaphor, and Lakoff \& Núñez [11] describe how these metaphors underlie mathematical thinking. In their terminology metaphor is not "simply a linguistic phenomenon, a mere figure of speech" (ibid p. 6). Rather, a conceptual metaphor is a cognitive mechanism that is - in technical terms - a "grounded inference-preserving cross-domain mapping" (ibid p. 6). Cross-domain mapping means that the mechanism applies a cognitive domain (e.g. motion) to reason about another domain (e.g. time). Because of inference-preservation we, for example, tend to think of time as going slow or fast. Grounding metaphors use our everyday experience of perception and action, like addition as adding objects to a collection. Linking metaphors use metaphorical ideas of one domain to reason about another domain. We use a linking metaphor, for example, when we think of numbers as points on a line.

Lakoff \& Núñez [11] present four grounding metaphors for arithmetic that include a conceptualisation of numbers. The numbers are conceptualised as collections of objects, as objects of different size, as segments of a measuring stick, and as point-locations on a path. Through linking metaphors numbers can also be conceptualised in terms of geometry (points on a line), symbols (sequences of numerals) and set theory (elements in a set). Different metaphors allow us different extensions of number system beyond the natural numbers. For example, the metaphor of numbers as locations on a path or a line, leads us easily to conceptualise negative numbers as locations left from the origin.

\section{Metaphors for infinity}

Our brains are finite, and our everyday experience consists solely of finite processes. Lakoff \& Núñez [11] have tried to answer how we yet can conceptualise infinity. The conceptual metaphor of infinity is embodied in the neural system that controls movement - the motor control system. This neural structure is not used only for motor control, but it can be used also to reason about events and actions. We conceptualise some actions to include their end ('to jump') and other actions to have their completion external to the act ('to fly'). Potential infinity is conceptualised through iterative processes that may be repeated on and on, and the completion of this process is external to the act. We can count numbers on and on, and we know that there is no boundary for the counting process.

However, when we use The Basic Metaphor of Infinity ([11, p. 159]), we take this iterative process that has no end, and metaphorically think of it in terms of a process that includes its completion and has a resulting state. This is a paradoxical thought - a process that goes on and on, and yet has a final state. 


$$
\text { "hannula" — 2007/2/15 - 13:51 — page } 322 \text { — \#6 }
$$

Using this metaphor for natural numbers, we can conceptualise the process of counting to be completed and we have the set of all natural numbers. We can also conceptualise 'the final resultant state' of counting, $\infty$, which is unique and bigger than all integers. Actual infinity " $\infty$ " cannot be conceptualised through potentially infinite process alone, but it requires the conceptualisation of an end point of the process [11].

Potential infinity can be used also to construct an unlimited number of decimal numbers between any given two numbers. However, actual infinity is required to conceptualise the size of the set as infinite. The difference between potential and actual infinity becomes crucial in the understanding of the statement $0.9999 \ldots=1$. When the number $0.999 \ldots$ is conceptualised as potentially infinite, it differs from one although the difference decreases as the process of adding new decimals continues. However, when it is conceptualised as actually infinite, the difference has disappeared as the end result of the process and therefore $0.999 \ldots$ equals one.

The metaphors for numbers and infinity are not arbitrarily chosen, but restricted by two main conditions. Firstly, they have plausible ultimate embodied groundings and evidence for it (e.g. cross-linguistic studies and spontaneous gestures). For example, humans have an innate capacity to subitize - to instantly recognize the number of up to three items. Cognitive capacities needed in order to count beyond three objects include grouping, ordering, pairing, memory, and exhaustion detection. Secondly, the embodied groundings

"must have just the right inferential structure and the hypothesized metaphors must have just the right mapping structure to account all the relevant mathematical inferences and all of the properties of the branch of mathematics studied" ([11, p. 101]).

If we accept the fundamental nature of conceptual metaphors in human thinking, then learning new mathematical ideas can be understood as a change of metaphor to be used for reasoning in one domain. For example, the learning of rational numbers can be seen as a shift from a conceptual metaphor of numbers as 'collections of objects' into a metaphor of numbers as 'points on a line'. Furthermore, in order to conceptualise real numbers there is need to apply other metaphors, like irrational numbers as infinite sequences of numerals.

In a study of the $5^{\text {th }}$ graders, Boero, Douek \& Garuti [1] distinguished three source domains for metaphors used in argumentation about infinity: another mathematical domain (typically geometry), everyday life experiences (a mortal woman giving birth to girl who will grow into a woman who will give birth to a 


$$
\text { "hannula" — 2007/2/15 — 13:51 — page 323 — \#7 }
$$

girl etc. thus producing infinitely long life), and religious ideas (eternity of God and/or soul).

\section{Focus of the paper}

We want to find out what is the level of students' understanding on infinity in Finnish comprehensive school, and how this understanding develops from grade 5 to grade 7 . We will distinguish three levels of students understanding of infinity. The lowest level is when they do not understand infinity, but use only finite numbers. In the intermediate level, the students understand potential infinity, and use processes that have no end. Those students, who have reached the third level, are able to conceptualise actual infinity and the final resultant state of the infinite process.

\section{Methods}

The paper describes some partial results of the research project "Development of Understanding and Self-confidence in Mathematics", implemented at the University of Turku (Finland), directed by professor Erkki Pehkonen, and financially supported by the Academy of Finland (project \#51019). The project was a two-year longitudinal investigation during the academic years 2001-03 on grades 5-8. More results of the project are to be found in the papers [10] and [12].

In order to measure the level of students' self-confidence and understanding of number concept in grades 5 and 7 of the Finnish comprehensive school, we designed a survey. The representative random sample of students consisted of 1154 fifth-graders (11 to 12 years of age) and 1902 seventh-graders (13 to 14 years of age) from different parts of Finland. The response rate of schools was $72 \%$. The survey was administered by teachers during a normal 45-minute lesson in the fall 2001. The questionnaire consisted of five parts: student background, 19 mathematics tasks, success expectation for each task, solution confidence for each task, and a mathematical belief scale.

The students' confidence was measured with three different measures. Before the students did the actual tasks, they estimated on a 5-point Likert scale whether they think that they can solve the task or not ('success expectation'). After solving each task they answered on another 5-point Likert scale on their confidence on their solution ('solution confidence'). In addition, we had a mathematical belief 


$$
\text { "hannula" — 2007/2/15 — 13:51 — page } 324 \text { — \#8 }
$$

scale with 25 items of which 10 were measuring students' self-confidence (adopted from Fennema-Sherman mathematics attitude scales [6]).

In the 19 mathematical questions, there were three that measured students' understanding of infinity (tasks 5, 7 and 8). Task 5 measured understanding of infinitely large natural numbers. The two other tasks measured understanding of the density of the rational numbers.

Task 5. Write the largest number that exists. How do you know that it is the largest?

Task \%. How many numbers are there between numbers 0.8 and 1.1 ?

Task 8. Which is the largest of numbers still smaller than one? How much does it differ from one?

Here we will concentrate on the results of these three infinity tasks.

After the survey was completed, five classes from each grade were selected, i.e. five grade 5 and five grade 7 classes. Furthermore, 40 students were selected to the qualitative part of the study, 20 students from each grade in question. The 40 students consisted of four students from each of the five grade 5 classes and of the five grade 7 classes. The students were interviewed, and they did some mathematical tasks in a group. In these situations, we returned to question 7 of the survey. This was done in order to get deeper insight of students' thinking.

\section{Results}

Survey results of competence

We categorized student responses to the infinity tasks according to how proper we deemed answers to be. In each question, we can find answers that remain on the level of finite numbers, answers that describe processes that do not end (potential infinity) as well as some answers that indicate that the student has an understanding of a final state of the infinite process (actual infinity).

To give a general description of the development from fifth grade to seventh grade we compared the answer distributions in each item. In Figures 1-3 we can see, that tasks were demanding and most students scored only zero or one point per task (maximum being 4-5 points). As expected, seventh graders gave better answers. 
Task 5. Write the largest number that exists. How do you know that it is the largest? Answer categories (and scoring):

- Actual infinity 2: There is no largest number (4 points)

- Actual infinity 1: Infinity, $\infty$ (3 points)

- Potential infinity: Unending number, e.g. 9999 ... (2 points)

- Finite: A number larger than one million, e.g. 99999999999999999, centillion (1 point)

- Incorrect: No answer or a number not exceeding one million (0 points)

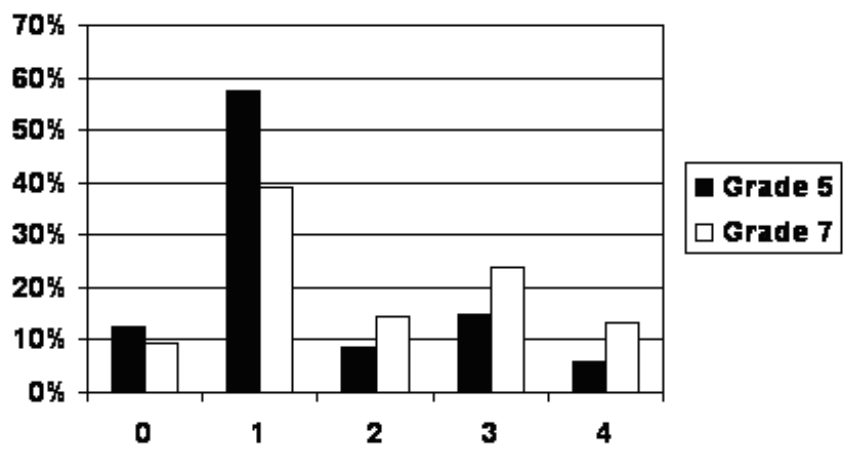

Figure 1. Students' scoring for task 5. Frequency of each answer category in grades 5 and 7

In task 5 (infinitely large), the development consisted mainly of the decrease of finite numbers as answers and of increase of different types of infinite answers.

Task 7. How many numbers are there between the numbers 0.8 and 1.1?

Answer categories (and scoring):

- Actual infinity: Infinitely many (5 points)

- Potential infinity: Unending number, e.g. 9999 ... (4 points)

- Finite 3: A finite number larger than one million, e.g. 9999999999999 (3 points)

- Finite 2: Working with more than one decimal, a number between 20 and one million (2 points)

- Finite 1: Working on one decimal level (even incorrectly), 2, 3 or 4 (1 point)

- Incorrect: Working on integral level (i.e. 1), no answer or other incorrect answers (e.g. 0.3) (0 points) 


$$
\text { "hannula" — 2007/2/15 — 13:51 — page 326 — \#10 }
$$

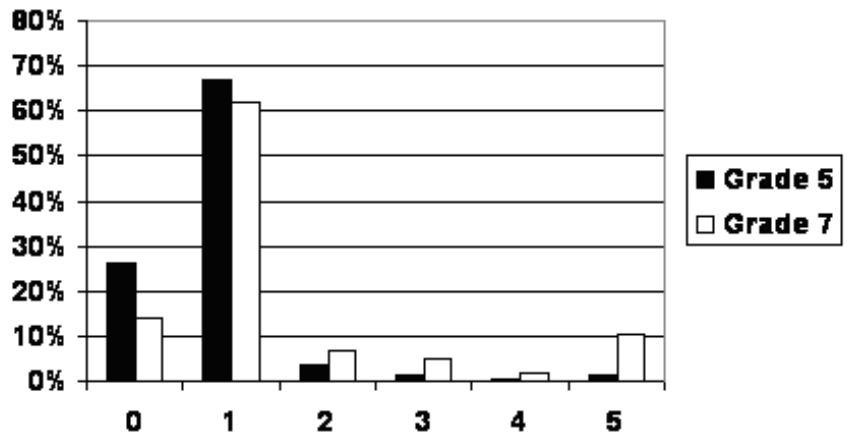

Figure 2. Students' scoring for task 7. Frequency of each answer category in grades 5 and 7

In task 7 (infinitely many), the decrease was mainly in completely incorrect answers (typically 0.3) and in single decimal thinking, and the biggest increase was in correct answers (infinitely many).

Task 8. Which is the largest of numbers still smaller than one? How much does it differ from one?

Answer categories (and scoring):

- Actual infinity: There is no such number (5 points)

- Potential infinity 2: Such number cannot be written (4 points)

- Potential infinity 1: $0.999 \ldots$ (3 points)

- Finite 2: 0.999; three or more decimals (2 points)

- Finite 1: 0.9; 0.99 (1 point)

- Incorrect: Working on natural number level (i.e. 0), no answer or other incorrect answers (e.g. negative infinity) (0 points)

In task 8 (infinitely close), the decrease was mainly in completely incorrect answers (typically 'zero' or 'minus infinity'), and a significant increase was in answers $(0.999 \ldots)$ that require understanding of potential infinity, but not actual infinity.

The chi square test revealed significant gender differences in task 5 (infinitely large) on fifth grade, and in tasks 7 (infinitely many) and task 8 (infinitely close) on seventh grade, in all cases boys giving significantly more frequently answers of infinite nature than girls. 


$$
\text { "hannula" — 2007/2/15 — 13:51 — page 327 — \#11 }
$$

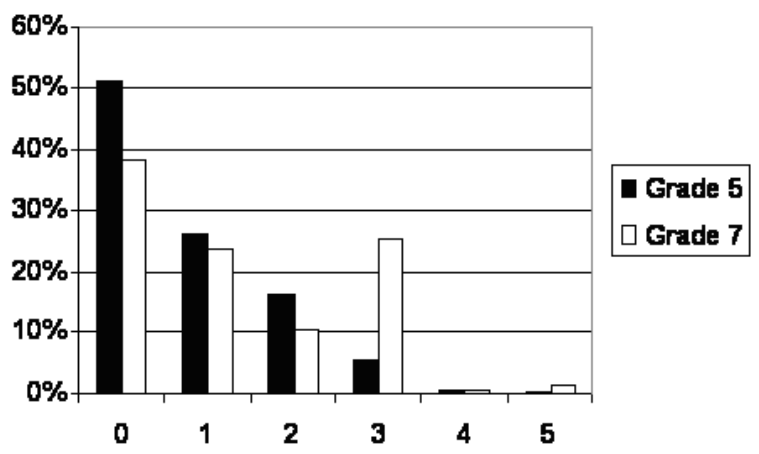

Figure 3. Students' scoring for task 8. Frequency of each answer category in grades 5 and 7

\section{Summary of competence results}

In the fifth grade 20 percent of the students had some understanding of the infinity of natural numbers, but few had any understanding of density of numbers. The situation is not much better in the seventh grade. Yet, there is an obvious development from grade 5 to grade 7 in student levels of answering these questions. Infinity of natural numbers is understood earlier than infinity of subsets rational (real) numbers, and potential infinity is understood earlier than actual infinity. Boys perform much better than girls in these tasks dealing with infinity.

\section{Survey results of confidence}

Next we shall look at how student's expectation of success and confidence in own solution relate to actual performance. As a general tendency students tended to use the upper end of both these scales indicating that most students had reasons for their answer and the results were not randomly picked. In earlier analyses it was observed that both average success expectation and average solution confidence had a moderate correlation with overall mathematical self-confidence $(0.49$ and 0.50 , respectively), while correlation with overall achievement in test was lower ( 0.33 and 0.40 , respectively). Below we shall report more detailed analyses on task-level.

According to the chi square test both students' success expectation and solution confidence related to their answers (with an exception of the fifth grade boys' success expectation). In tasks 5 and 8 , the students' solution confidence 
increased, as their answers got better. In task 7 (infinitely many), however, the relationship between answer and confidence was more complex (Table 1). Students who gave 0- or 1-point answers were modestly uncertain, while solution confidence was much lower for 2-point answers. Confidence remained low for 3and 4-point answers and was high for 5-point answers. Students who operate on one decimal level seem to be confident on their answers, while those more advanced students who move beyond that level have lower confidence. Only when they realize that there are infinitely many numbers within the given interval, they regain high confidence.

Table 1. The means of solution confidence for responses of task 7

\begin{tabular}{|c|c|c|c|c|c|c|}
\hline $\begin{array}{c}\text { Points } \\
\text { for task } \\
7\end{array}$ & $N$ & $\begin{array}{c}\text { Success } \\
\text { expectation } \\
\text { mean }\end{array}$ & $\begin{array}{c}\text { Std. } \\
\text { deviation }\end{array}$ & $N$ & $\begin{array}{c}\text { Solution } \\
\text { confidence } \\
\text { mean }\end{array}$ & $\begin{array}{c}\text { Std. } \\
\text { deviation }\end{array}$ \\
\hline 0 & 561 & 4.06 & 1.06 & 539 & 3.42 & 1.35 \\
\hline 1 & 1933 & 4.15 & 0.94 & 1922 & 3.68 & 1.16 \\
\hline 2 & 171 & 3.99 & 0.94 & 169 & 2.91 & 1.19 \\
\hline 3 & 109 & 3.54 & 1.28 & 104 & 3.10 & 1.56 \\
\hline 4 & 42 & 3.88 & 1.11 & 40 & 3.18 & 1.52 \\
\hline 5 & 210 & 4.07 & 1.16 & 210 & 3.92 & 1.12 \\
\hline Total & 3026 & 4.09 & 1.00 & 2984 & 3.58 & 1.24 \\
\hline
\end{tabular}

Looking at the results on an individual level (not presented here), we can see that most typical relationship between success expectation and solution confidence is that they are equal. However, among students who gave two-point answers most were less confident than their expectation had been, and there were hardly any students whose confidence after the task was higher than the expectation they had before solving it. We can assume, that most students who moved beyond 'one decimal thinking', had not realized the complexity of the task before actually beginning to solve it. Among students who gave three-point answers, the pattern was similar, although less extreme.

The relationship between answer and its success expectation was slightly different from the relationship between answer and its solution confidence presented above. For task 5 (infinitely large) those students who gave 3-point answers ("infinity") had highest expectations, for task 8 (infinitely close) expectations were highest when the answer got 2 or 3 points ("0.999", three or more decimals or 


$$
\text { "hannula" — 2007/2/15 — 13:51 — page 329 — \#13 }
$$

"0.999 ...", respectively). This suggests that those who gave the best answers did not know the right answer beforehand, but they had to produce it during the test. Furthermore, for task 7 (infinitely many) only those students who gave 3-point answers (a large finite number) had much lower expectations than others. Especially those students who gave a 2-point answer (20 - one million) had roughly as high expectations as others (cf. Figure 4).

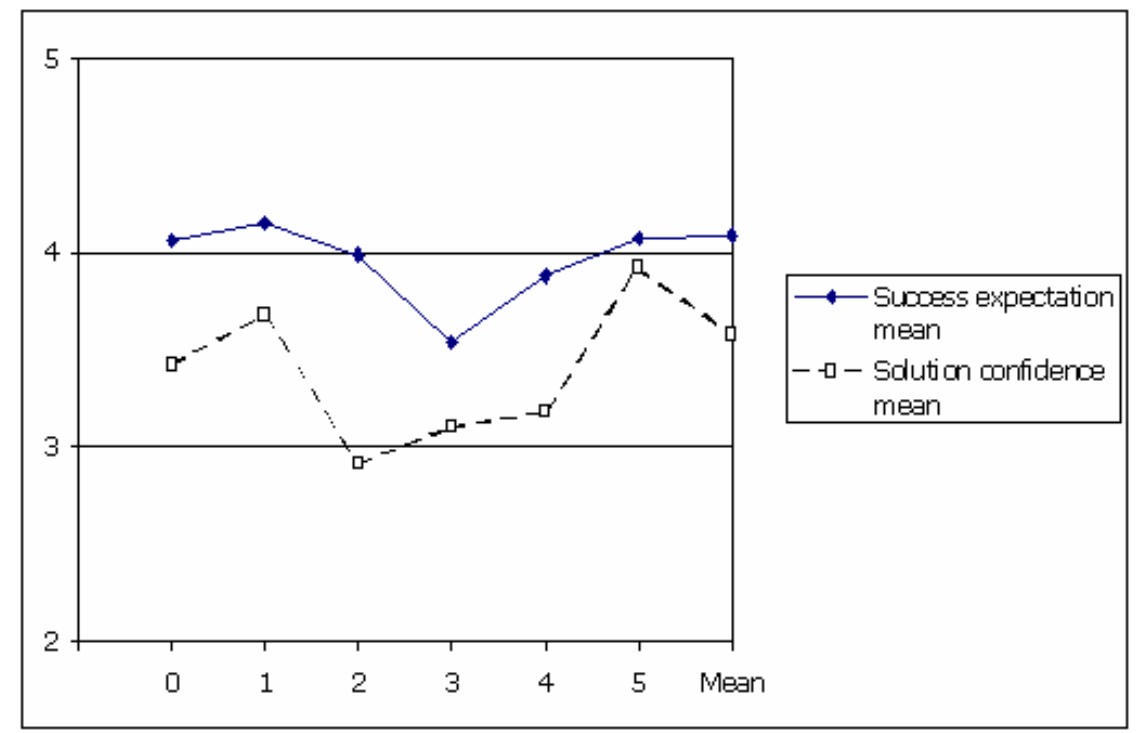

Figure 4. Comparison of the means in success expectation and in solution confidence in different levels of solutions (Task 7)

In all cases, the students' success expectation was higher than their solution confidence. In the result group 2 (Working with more than one decimal, a number between 20 and one million), the difference was the biggest one, and in the best answers (group 5) the smallest one.

\section{Summary of confidence results}

Students' confidence both before and after solving the task is related to the success they have. That is what we should expect to find. However, those who gave the most sophisticated answers were not the most confident in their expectations. 


$$
\text { "hannula" — 2007/2/15 — 13:51 — page 330 — \#14 }
$$

In the task (task 7) where we asked how many numbers there are between 0.8 and 1.1, students' confidence had even more complex relationship with success. Many of the students indicated strong false confidence in their one-decimal thinking of numbers. Furthermore, when their thinking begun to advance, their confidence dropped. Sometimes they even had an initial expectation of success before they begun to solve the task but this confidence fell after they had tried to solve the task. Confidence was reassured when they reached the level where they had an understanding of the density of numbers.

\section{Qualitative results}

In the qualitative part of the study, students were asked the same question about the number of numbers between 0.8 and 1.1 as in the test, and they were also asked to explain their reasoning. One of the students (grade 7) simply knew immediately that there are infinitely many numbers. He was also able to reason why this was the case. He was the only one to reason with actual infinity.

From the students' explanations we found four interesting phenomena that we shall describe below: (1) transformation of the decimal numbers into whole numbers, (2) ability to find more numbers within the interval when further prompted, (3) assumed finiteness of a bounded interval, (4) reasoning based on the concretely drawn number line.

(1) Some students talked about whole numbers 9, 10 and 11. To our understanding, this reflects that they were reasoning about the decimals as natural numbers. Cf. the following episodes from the $5^{\text {th }}$ grade $_{\text {classes }}{ }^{1}$

S1: Thrrree. Three.

S2: Four. 8, 9, 0, 1.

S4: I know, three.

S6: Three.

I: What do you others think??

S7: Three.

S8: $\{$ Nods $\}$.

S6: 9, 10, 11.. But no, 2: 9 and 10.

I: Well how many numbers do you think that there are then?

S8: In between you mean?

\footnotetext{
${ }^{1}$ In the transcripts the following conventions are used: [text in square brackets indicates overlapping speech]; "<" marks an interrupted flow of speech; text in ellipses indicates nonverbal information
} 


$$
\text { "hannula" — 2007/2/15 — 13:51 — page 331 — \#15 }
$$

I: Yes.

S8: 2.

I: Well, what numbers are there then?

S6: 9 and 10.

S8: 0.9 and 1.0 .

Others agree.

(2) Several $5^{\text {th }}$ graders thought first on decimal level and suggested only two, three or four numbers. However, when they were further prompted, some students either suggested isolated numbers ("1.05"; "1.09") or answered that there are also hundredths and thousandths. Some other students thought of fractions ("fractions of fractions of fractions") as existing between decimals. However, only one $5^{\text {th }}$ grader even pondered the possibility of infinitely many numbers.

S6: Well, fractions at least and then they decrease into fractions of fractions of fractions and so on. And then they become smaller all the time.

I: How small can they become?

S6: I don't know how small they can become.

Similar thinking was common even on the $7^{\text {th }}$ grade.

S21: There could be hundredths, too.

I: Give an example.

S21: Well, for example 0.81.

I: And anything else?

S22: Thousandths.

I: What are they like?

S22: Well, for example 0.938.

(3) There was one clear example of mistaking boundedness to be the same as finiteness. One seventh-grader (S25) refused to accept her peers' reasoning that there would be infinitely many numbers within a bounded interval of the number line.

S25: that there are almost infinite.

S26: $\{$ whispers to S27\} (five)

S28: (Well, there) is that infinity. It can't be, in a way, almost infinity, because it, when [isn't $<$ (its not $\leq)$ ].

S25: [Yes it can.] Think about it. Infinity is so, that it doesn't end [ever, you know.] 


$$
\text { "hannula" — 2007/2/15 — 13:51 — page 332 — \#16 }
$$

S28: [That's not a number.]

S25: But because there are two numbers there, and in-between them, so it will end some time.

S27: No it doesn't.

S26: It does not end. 0.8888 blablabla

S27: 0.00[00000]

S25: [Well, yes, but] it will end where is the point \{points to the number 1.1 \} where it has to end.

(4) Students sometimes reasoned about the task using a mental image of a number line. In one interview seventh grade students also expressed views that indicate that they draw inferences from the properties of concretely drawn number lines - specifically that each number needs some space.

S29: There would be at least $m<$ at least more $<$ in principle more $<$ must be 20000 numbers, maybe.

S30: But those all cannot fit into there!

S29: Well, yes.

S30: Well, of course in a way.

S29: In a way, but one could no way squeeze them into a number line like this.

\section{Summarizing results from interview data}

There was quite clear evidence that at least some students use natural numbers to reason about decimal numbers. Some even erroneously talk about whole numbers instead of decimal numbers. Although most students initially answered that there was two or three numbers between 0.8 and 1.1 , many were able to produce further numbers when prompted to reconsider.

There was also evidence for blending of concrete and abstract number line models. Some reasoned that infinitely many numbers cannot 'fit' within a bounded interval. Based on our results, we suggest that students who answered that there was more than three numbers (but not infinitely many), had an intuition that they had not found the end of the process, and therefore their solution confidence was low. Furthermore, when they first had read the task, their success expectation had been high. This suggests that these students realized that they need to move beyond the one decimal level while they were solving the task. In order to understand that there are infinitely many numbers between 0.8 and 1.1 , the students needed to realize that the process of constructing more and more numbers within the given interval is potentially infinite. Furthermore, they need to 


$$
\text { "hannula" — 2007/2/15 — 13:51 — page 333 — \#17 }
$$

use the basic metaphor of infinity to conceptualise the final resultant state of the process - infinitely many numbers. Only when they would reach that level they would feel confident.

Most students who understood that there are infinitely many numbers within the interval seemed to find that solution through reasoning a potentially infinite process. Only one student immediately reasoned with actual infinity. We might say that the former represents an approach of constructing a solution through a process, while the latter represents deduction from a known property (density) of a number line.

\section{Conclusions}

\section{Discussion}

In the fifth grade most students have no clue of infinity, and the situation is not much better in the seventh grade. Yet, there is an obvious development from grade 5 to grade 7 in student levels of answering these questions. Infinity is, indeed, a difficult concept and students have difficulties with it, even in high school [13] and at the university level [15].

In international comparative studies the gender difference in mathematics achievement among 15-year olds has disappeared in many countries, Finland being one of those countries [17], [18]. However, in this representative large sample boys gave significantly better answers than girls in tasks dealing with infinity. This finding can be understood in the light of the general conclusion made by Fennema and Hart [5]. According to them, gender differences in mathematics still remain within the most difficult topics. The test used can be regarded as an example of a very challenging one that is likely to produce large gender differences even in countries where overall gender differences in mathematics achievement have disappeared.

In most cases students who gave better answers were also more confident of their answers. This is what we would have expected. However, findings for task 7 confront this expected tendency. Also Merenluoto [13] has found similar results, where there was a general tendency for confidence to increase as the answers got better, but also some topics where this was not the case.

Our empirical evidence supports some of the ideas we presented in the beginning. The nature of non-linearity in students' solution confidence supports the 


$$
\text { "hannula" — 2007/2/15 — 13:51 — page 334 — \#18 }
$$

view that learning infinity requires a conceptual change. Furthermore, our interview data supports the view that many students reason about decimal numbers with a linking metaphor to natural numbers. Students who work on a single decimal level are confident of their answer. They have assimilated tenths into their concept of natural numbers. When students realise that it is possible to generate more decimals, hundredths, thousandths, etc., it is like opening a Pandora's box. The logic of natural numbers is challenged: is the successor of $0.80 .9,0.81$, or 0.80001 ? Even if they still produce a finite answer, they are uncertain of their answers, because they are aware of the alternatives. Only when their conception of (rational) numbers has changed to include the idea of density, can they again gain confidence.

Regarding metaphors, we found clear evidence that students used natural numbers to reason about decimal numbers. Another metaphor that we found evidence for was the number line metaphor. At least some students draw from this metaphor the incorrect inference that each number would occupy a space on the number line. However, unlike in the study by Boero et al. [1] we found no reference to God or chain of generations as source metaphors for infinity. This suggests that some metaphors are culture-dependent.

\section{Some final comments}

Mathematics is often considered to form a hierarchical structure where all new concepts logically follow from prior ones, which allows students to enrich their knowledge step by step. This hierarchy of structures was most clearly seen in the bourbakist way of compiling mathematics according the structures (cf. [2]). Such a structural way of learning mathematics can, however, be dangerous, since in some cases old structures may disturb the adaptation of new and more general ones. The very fundamental idea of a successor, for example, is necessary for learning the notion of natural numbers. The idea is, however, later on seriously conflicting with the understanding of the character of both rational and real numbers. According to the theories on conceptual change (cf. [3], [23], [14]) the relationship between learners' prior knowledge and new information to be learned is one of the most crucial factors in determining the quality of learning.

Theories of conceptual change focus especially to those processes where an earlier way of thinking is challenged by new knowledge to be learned (e.g. [13], [14]). Since learning can happen, at least, in two different ways - using the Piagetian language: through accommodation or through adaptation - we have also two categories of conceptual change theories. Enrichment (cf. assimilation) is 


$$
\text { "hannula" — 2007/2/15 — 13:51 — page 335 — \#19 }
$$

an easier process, where old knowledge remains mainly valid, and new knowledge is added to the slightly modified knowledge structure. The second category of conceptual change (cf. accommodation) is a more complicated one, since it needs a drastic revision or restructuring of old knowledge. Moving from potential infinity to actual infinity of natural numbers is an example of enrichment, and some students can handle this already at grade five. A more radical restructuring is necessary for grasping the density of rational (real) numbers. It requires moving beyond the logic of natural numbers where each number has a successor and this seems to be more difficult for students. In another analysis of the longitudinal development of student competence in number concept, we noticed that proper understanding of fractions as numbers is an important predictor of learning the density of numbers [9]. This suggests that learning fractions is an important opportunity for this challenging conceptual change.

In our opinion the results are not satisfactory. Majority of students seemed to think purely on the level of finite processes. The basic idea of potential infinity is not difficult to introduce to students. Usually children are very interested about these questions. Are teachers afraid of this topic as too difficult? In school we should teach mathematics and not only to master routine tasks of the textbook. This means that the main mathematical ideas should be discussed in the class, too. Infinity is one of the mathematical 'Fundamental Ideas' that need to be introduced to children early on [21]. That infinity is introduced relatively late in the curriculum, may be especially harmful for female students, who tend to rely more on the ideas taught in school. Students need experiences that allow them to develop rich images of the topic, which will function as the basis for a formalisation at a later stage [20].

\section{References}

[1] P. Boero, N. Douek, R. Garuti, Children's conceptions of infinity of numbers in a fifth grade discussion context, in: Proceedings of the $27^{\text {th }}$ Conference of the International Group for the Psychology of Mathematics Education, Vol. 2, (N. A. Pateman, B. J. Dougherty, J. Zilliox, eds.), University of Hawaii, 2003, 121-128.

[2] C. B. Boyer, A history of mathematics, Princeton University Press, Princeton (NJ), 1985.

[3] S. Carey, Conceptual change in childhood, MIT Press, Cambridge, MA, 1985.

[4] P. J. Davis, R. Hersh, The Mathematical Experience, Birkhäuser, Boston, MA, $1980 / 1990$. 


$$
\text { "hannula" — 2007/2/15 — 13:51 — page 336 — \#20 }
$$

[5] E. Fennema, L. E. Hart, Gender and the JRME, Journal for Research in Mathematics Education 24, no. 6 (1994), 648-659.

[6] E. Fennema, J. A. Sherman, Fennema-Sherman mathematics attitudes scales, JSAS Catalog of Selected Documents in Psychology 6, no. 31 (1976), Ms. No. 1225.

[7] E. Fischbein, Intuition in Science and Mathematics, Reidel, Dordrecht, The Netherlands, 1987.

[8] E. Fishbein, D. Tirosh, P. Hess, The intuition of infinity, Educational Studies in Mathematics 1 (1979), 3-40.

[9] M. S. Hannula, H. Maijala, E. Pehkonen, Development of understanding and self-confidence in mathematics; grades 5-8, in: Proceedings of the $28^{\text {th }}$ Conference of the International Group for the Psychology of Mathematics Education, Vol. 3, (M. J. Høines, A. B. Fuglestad, eds.), Bergen University College, 2004, 17-24.

[10] M. S. Hannula, H. Maijala, E. Pehkonen, A. Nurmi, Gender comparisons of pupils' self-confidence in mathematics learning, Nordic Studies in Mathematics Education 10, no. 3-4 (2005), 29-42.

[11] G. Lakoff, R. E. Núñes, Where mathematics comes from; How the embodied mind brings mathematics into being, Basic Books, 2000.

[12] H. Maijala, How does classroom teaching in mathematics look like?, manuscript, 2005.

[13] K. Merenluoto, Lukiolaisen reaaliluku; Lukualueen laajentaminen käsitteellisenä muutoksena matematiikassa (in English: Students' real number; Enlargement of the number concept as a conceptual change in mathematics), University of Turku, 2001, Publications C 176.

[14] K. Merenluoto, Discussion about conceptual change in mathematics, Nordic Studies in Mathematics Education 10, no. 2 (2005), 17-33.

[15] K. Merenluoto, E. Pehkonen, Elementary teacher students' mathematical understanding explained via conceptual change, in: Proceedings of the PME-NAXXIV, (D. Mewborne, P. Sztajn, D. Y. White, H. G. Wiegel, R. L. Bryant, K. Nooney, eds.), ERIC, Columbus (OH), 2002, 1936-1939.

[16] A. L. E. Moreno, G. Waldegg, The conceptual evolution of actual mathematical infinity, Educational Studies in Mathematics 22, no. 3 (1991), 211-231.

[17] I. V. S. Mullis, M. O. Martin, E. J. Gonzales, K. D. Gregory, R. A. Garden, K. M. O'Connor, S. J. Chostowski, T. A. Smith, TIMSS 1999 international mathematics report; Findings from IEA's repeat of the Third International Mathematis and Science Study at the eight grade, International Study Center, Lynch School of Education, Boston College, 2000.

[18] OECD/PISA, Problem solving for tomorrow's world; First measures of cross-cultural competencies from PISA 2003, OECD, 2004.

[19] J. Piaget, B. Inhelder, A child's conception of space, Routledge \& Kegan Paul, London, 1967. 
[20] S. Pirie, T. Kieren, Growth in mathematical understanding: How can we characterise it and how can we represent it?, Educational Studies in Mathematics 26, no. 2-3 (1994), 165-190.

[21] F. Schweiger, Fundamentale Ideen. Eine geistesgeschichtliche Studie zur Mathematikdidaktik, Journal für Mathematik-Didaktik 13 (1992), 199-214.

[22] P. Tsamir, T. Dreyfus, Comparing infinite sets - a process of abstraction. The case of Ben, The Journal of Mathematical Behavior 21, no. 1 (2002), 1-23.

[23] S. Vosniadou, Capturing and modeling the process of conceptual change, Learning and Instruction 4 (1994), 45-69.

[24] M. M. Wheeler, Children's understanding of zero and infinity, Arithmetic teacher (Nov 1987), 42-44.

[25] I. Wistedt, M. Martinsson, Orchestrating a mathematical theme: eleven-year olds discuss the problem of infinity, Learning and Instruction 6, no. 2 (1996), 173-185.

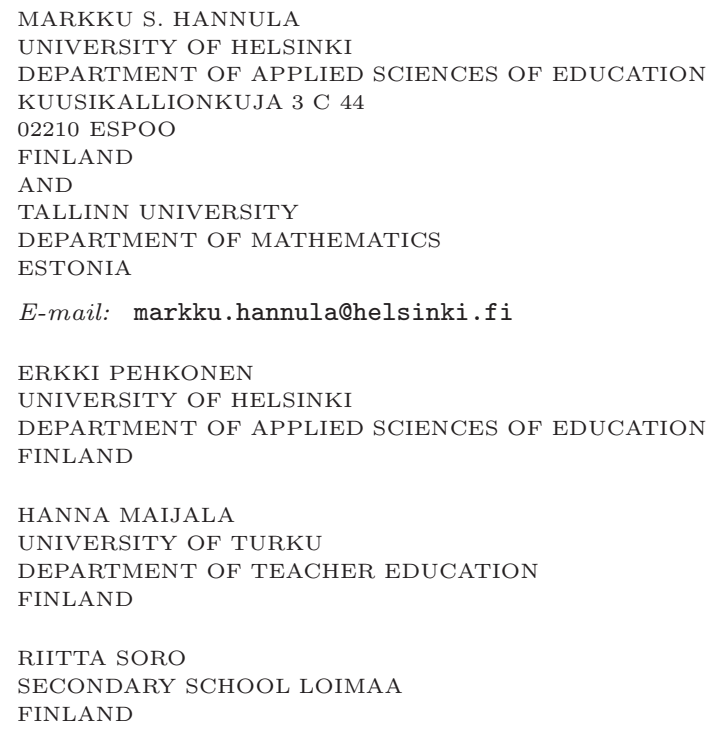

